\title{
Localization of premature ventricular contraction foci in normal individuals based on multichannel electrocardiogram signals processing
}

\author{
Sima Soheilykhah ${ }^{1}$, Ali Sheikhani ${ }^{2 *}$, Alireza Ghorbani Sharif ${ }^{3}$ and Mohammad M Daevaeiha ${ }^{4}$
}

\begin{abstract}
A premature ventricular contraction (PVC) is relatively a common event where the heartbeat is initiated by the other pathway rather than by the Sinoatrial node, the normal heartbeat initiator. Determining PVC foci is important for ablation procedure and it can help in pre-procedural planning and potentially may improve ablation outcome. In this study, 12-lead Electrocardiogram (ECG) of 87 patients without structural cardiac diseases, who had experienced PVC, were obtained. Initially, PVC foci were labeled based on Electrophysiology study (EPS) reports. PVC beats were detected by wavelet method and their foci were classified using Mahalanobis distance and One-way ANOVA. Using morphological, frequency and spectrogram features, these foci in the heart were classified into five groups: Left Ventricular Outflow Tract (LVOT), Right Ventricular Outflow Tract (RVOT) septum, basal Right Ventricular (RV), RVOT free-wall, and Aortic Cusp (AC).

The results showed that $88.4 \%$ of patients are classified correctly.
\end{abstract}

Keywords: Electrocardiogram; Premature ventricular beats; PVC foci (focuses)

\section{Introduction}

Premature ventricular contraction (PVC) is the most common cardiac arrhythmia in patients with or without any kind of diagnosed cardiac diseases (Chiu et al. 2005). PVC is an extra heart beat originates from the ventricles and comes before the normal heart beat. Although in general, this arrhythmia may occur in a healthy person, but it is mostly associated with elderly patients (males: 60-80\%) (Nathani et al. 2007) and patients suffering cardiac diseases such as hypertension, myocardial infarction, and so on (Chikh et al. 2010).

Today, the electrocardiogram (ECG) still remains the simplest and cost effective non-invasive diagnostic method for determining arrhythmias. Physicians interpret the morphology of the ECG waveform and decide whether the heartbeat belongs to the normal sinus rhythm or to the class of cardiac arrhythmias. Thus it is useful to localize the site of origin of PVCs before ablation procedure. This

\footnotetext{
* Correspondence: sheikhani_al_81@srbiau.ac.ir

${ }^{2}$ Assistant Professor of Department of Biomedical Engineering, Islamic Azad University, Science and Research branch, Tehran, Iran

Full list of author information is available at the end of the article
}

helps not only in pre-procedural planning, but also can potentially improve ablation outcomes (Lin et al. 2008).

PVCs may originate from various foci. If PVC focus is in right ventricle, it would appear as Left Bundle Branch Block (LBBB) and if it is in left ventricle, it would appear as Right Bundle Branch Block (RBBB) because in this state left ventricle would depolarize earlier. In general there are three common regions are defined for PVC foci: Right Ventricular Outflow Tract (RVOT), Left Ventricular Outflow Tract (LVOT) (Betensky et al. 2011) and Aortic Cusp (AC). Many researches for determining various divisions of idiopathic VT or PVC foci have been developed so far, including idiopathic ventricular tachycardia (IVT) consist of RVOT VT/PVC, Idiopathic Left Ventricular Tachycardia (ILVT), Idiopathic Propranolol sensitive VT (IPVT), LVOT VT/PVC (Nathani et al. 2007) and AC (Lin et al. 2008). It has been reported that $60-80 \%$ of the idiopathic tachycardia in normal hearts arise from the RVOT and 10\% of them arise from LVOT (Lerman et al. 1997). RVOT VT/PVC is more common in females at age 30 to 50 years old (Nakagawa et al. 2002) with wide QRS complex and LBBB pattern in inferior axis (Shin et al. 2007), whereas LVOT VT/PVC 
usually shows RBBB morphology in lead V1 with wide monophasic R-wave in precordial leads. Morphologic explanations of ECG characteristics are useful for differentiating of VT/PVC arising from the AC region. VT/ PVC originated from the left coronary cusp produces multiphasic QRS morphology with an M or W configuration in lead V1 with a precordial transition no later than V2. A left bundle pattern with a wide small $\mathrm{R}$ wave in lead V2 and a precordial transition usually at V3 is revealed in PVCs with a right coronary cusp origin (Lin et al. 2008).

Kamakura et al. (Kamakura et al. 1998) proposed the method to estimate the origin of VT/PVC from the RVOT and LVOT by using indexes obtained from 12-lead ECG. They classified PVC/VT from the RVOT into 8 subdivisions by using 3-dimensional anatomic relation: anteriorposterior, right-left, and superior-inferior. The features they used for estimating the origin of PVC/VT consisted of morphology, amplitude, duration and polarity of QRS complex. To distinguish LVOT from RVOT region, they showed that $R / S$ amplitude ratio in lead $V_{3}$ is a helpful index. If the ratio of $\mathrm{R} / \mathrm{S}$ amplitude in $\mathrm{V}_{3}$ is equal or higher than 1, the PVC/VT stems from LVOT zone, otherwise arises from RVOT.

The aim of this study is classifying PVCs and finding their foci using multichannel ECG signals processing and various features such as morphological, frequency and spectrogram features.

\section{Materials and methods Data collection}

12 lead ECGs of the 90 patients without structural cardiac disease, in whom PVC or VT had been ablated successfully, were used. The data was collected in Tehran Arrhythmia Clinic. The PVC foci were confirmed during the ablation procedure and attached as a label of data. Based on EPS reports, foci were divided into six subgroups including: LVOT (anterior \& posterior), RVOT septum, basal RV, RVOT free-wall, Aortic cusp (Left Coronary cusp, Right Coronary cusp \& NonCoronary cusp) and LV body.

According to the EPS reports, 8 out of all PVCs were originated from the LVOT. Of the 52 PVCs originating from the RVOT, 15 were grouped as of the free-wall origin, 37 of the septum zone. 18 of PVCs had originated from the aortic cusp. 3 of PVCs were arisen from the LV body. The origins of remained PVCs were identified as the basal RV. Due to the lack of patients whose PVCs originating in LV body (there were only 3 patients), this group was omitted and study was done with remaining 87 patients.

Figure 1 shows a sample of 12-lead ECG of patients with PVC originating from RVOT septum.

\section{PVC detection}

First of all, PVCs are recognized and distinguished from the normal beats. Because of their greatness in height, depth and length, PVCs could easily be detected. Since 12 leads ECG signals were recorded simultaneously, one lead was used to detect duration of the PVC beat and finally the PVC beats were extracted in all leads. Because PVC was more obvious than normal one in lead III, this lead was used for detecting PVC heartbeats.

In order to attenuate high frequency components, the ECG signal was filtered using a low pass equiripple finiteduration impulse response (FIR) filter with cut-off frequency at $25 \mathrm{~Hz}$ implemented with MATLAB 7.8.0 (R2009a) (The Mathworks, Inc.). In this way, considering lower frequency content of PVCs, beats would become more obvious than normal one and would be detected easier.

The standard parameters of the ECG waveform can be determined with high accuracy using wavelet transforms (Sumathi \& Sanavullah 2009; Bensegueni \& Bennia 2012). The wavelet transform (WT) provides a representation of the signal in time-scale domain, allowing representation of the temporal features of a signal at different resolutions. Detection of these main points is based on maximum absolute values and zero crossing of WTs at specific scales (Chang et al. 2013).

The Haar wavelet was used in our study at scale $2^{1}$ for PVC detection (Sumathi \& Sanavullah 2009). R peak in PVC beat is detected by marking the zero crossing of the WT between positive maximum-negative minimum pair (Martínez et al. 2004). Ascendant edge of the wave is relevant to negative minimum and decreasing edge of wave is relevant to positive maximum.

First we chose a peak greater than the threshold (0.1) and then absolute of WT signal was calculated. Then we determined the nearest peaks before and after the chosen peak and finally selected the greater one. The first minimum before the initial peak and the first minimum after the second peak were specified as onset and offset of PVC beat. Because of simultaneous recording of ECG leads, duration of onset and offset of PVC beat was assigned to other leads and PVCs were determined in all leads.

\section{Features extraction}

In this study we extracted the morphological, frequency and spectrogram features after PVCs detection to classify their five foci. Since a physician classifies arrhythmia with the information of rhythm and morphology, an input vector can consist of features that illustrate the rhythm and morphology properly (Song et al. 2005).

One of the morphological features is polarity of eachlead ECG signal. The positive, biphasic or negative polarity was considered with 1, 0, or -1 respectively. Existence or 

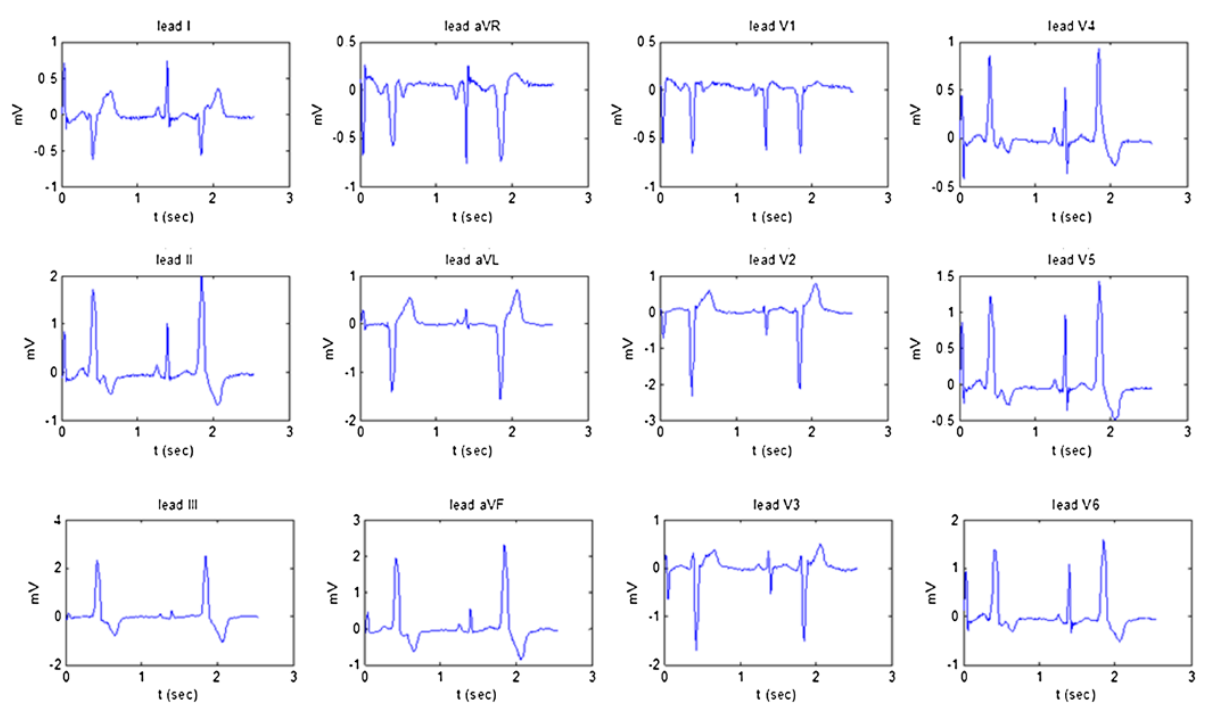

Figure 1 A sample of 12-lead ECG signal of a patient with PVC originated from RVOT septum.

absence of notching (Yamashina et al. 2011) in signal was shown with 1 or 0 . Notching in QRS complex is determined as a tri-phasic $\mathrm{R}$ or $\mathrm{Q}$ wave with an interval greater than $40 \mathrm{~m}$-sec between the first and second peak of the QRS complex. Existence of notching is considered when notching is observed in more than three of the six limb leads. As it was obtained, notching was observed more often in the PVCs arising from the free-wall rather than in the PVCs originating from the septal region (Tada et al. 2007). Figure 2 shows a sample of notched QRS complex.
Spectrum content of ECG signal in various leads is another feature that is used in classification of PVCs in this study. Frequency band of $\mathrm{P}$ and $\mathrm{T}$ waves in lead II, the most applicable lead, is approximately 0.5 to $10 \mathrm{~Hz}$ and QRS complex has frequencies ranging approximately from 3 to $40 \mathrm{~Hz}$. Heart rate dependencies of waves in ECG must be taken into consideration. Heart rate changes will cause changes in waveforms and frequency contents. So some frequency components of ECG signal can be defined as classifying features and Fast Fourier Transform (FFT)

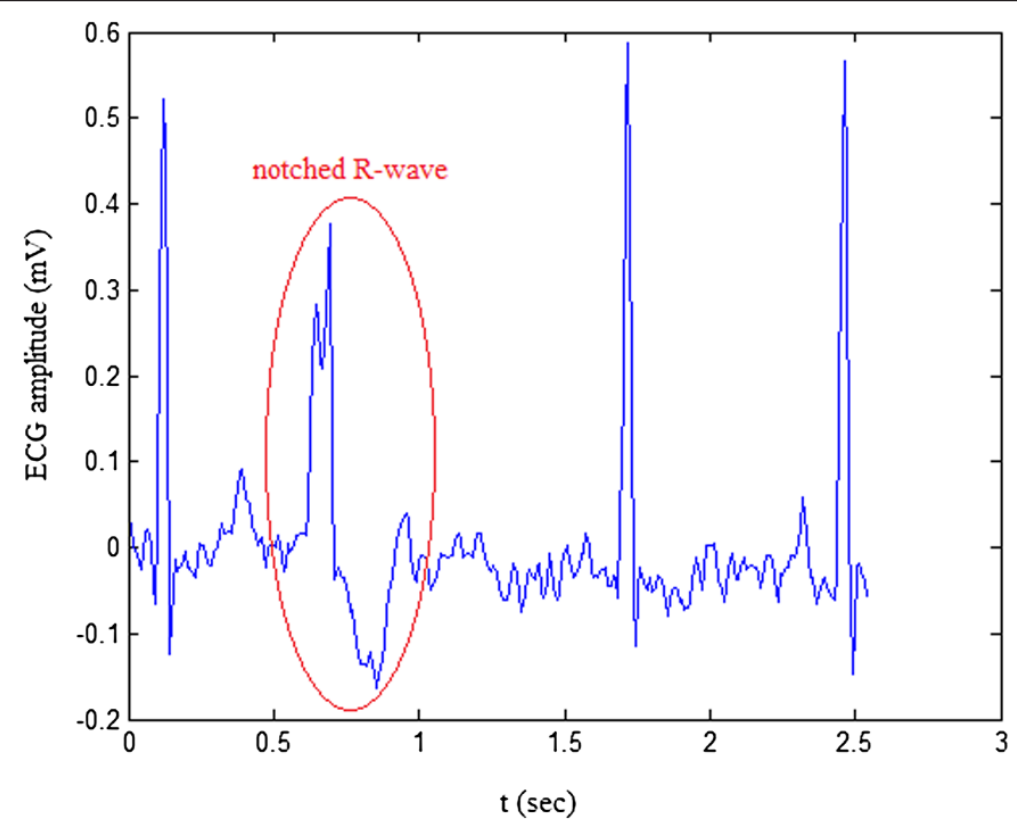

Figure 2 A sample of notched QRS complex. 
was used for this purpose. Fourier transform of a signal and its inverse are calculated by following equations (Subha et al. 2010).

$$
\begin{aligned}
& X(f)=\int_{-\infty}^{\infty} x(t) e^{-j 2 \pi f t} d t \\
& x(t)=\int_{-\infty}^{\infty} X(f) e^{j 2 \pi f t} d f
\end{aligned}
$$

In these equations, $t$ represents time and $f$ denotes frequency, $x(t)$ is original signal in time domain and $X(f)$ represents its Fourier transform in frequency domain.

The Short-time Fourier transform (STFT) is obtained from the Fourier transform by multiplying the time signal $x(t)$ by a window function (Tokmakei \& Erdogan 2009; Hardalac et al. 2007).

$$
\operatorname{STFT}(\omega, \tau)=\int_{-\infty}^{+\infty} x(t) \Psi^{*}(t-\tau) e^{-j \omega \tau} d t
$$

Spectrogram, magnitude of STFT, shows frequency characteristics of signals in the time domain. The average of spectrogram greater than the 70 percent (Sheikkani et al. 2012) was computed for all leads and used as a feature for separating the groups.

The other spectral features are as follows: Maximum amplitude of spectrum signals, variance of signals' spectrum, average of FFT of signal, power spectrum and power spectrum bandwidth of signals, the average of amplitudes of spectrum signals greater than the 70 percent and the average of Haar wavelet coefficients at level 1 and 2.

\section{Statistical analysis and classification}

Since the assumptions of normal distribution and similarities were valid, statistical analyses of One-Way ANOVA were performed in SPSS 17.0 software to evaluate differences of features. The $\mathrm{p}<0.05$ was considered to show significant differences. Mahalanobis distance (Yeh 2009) was used for classifying the five groups by considering morphological, spectrogram and frequency features.

To determine the separation ability of pairwise groups, we used SVM classifier in MATLAB. Support Vector Machines (SVM) are supervised learning models with associated learning algorithm that analyze data and recognize patterns, used for classification. The basic SVM takes a set of input data and predicts, for each given input, which of two possible classes forms the output. It is more considerable using SVM classifier than Mahalanobis distance to discriminate two groups. We set data and their label of each group in separate matrixes, defined train and test data, then classified two groups each time and computed errors and compared them.

\section{Results}

The PVC beats were extracted from 12-lead ECG signals using the wavelet. Figure 3 shows one lead of ECG signal and extracted PVC relevant to a sample of the patients whose PVC arose from RVOT septum.

Using one-way ANOVA, the $p$-values of the 12 leads of ECG features between five groups, consist of LVOT, RVOT septum, basal RV, RVOT free-wall and Coronary cusp, are shown in Table 1. It was observed that wavelet features had no significant differences; however morphological features (QRS polarity) showed more significant differences than other features. The leads aVL, V2 and V3 showed the least significant differences, as illustrated in Table 1.

By considering all the features, morphological, wavelet, frequency and spectrogram, the best classification result are achieved by $88.4 \%$ (with 0.6667 and 0.9305 sensitivity and specificity, respectively) using Mahalanobis distance. The result of classification with only the frequency features was obtained $68.1 \%$, with spectrogram features alone and with only morphological features were $55.1 \%$ and $84.1 \%$ respectively.
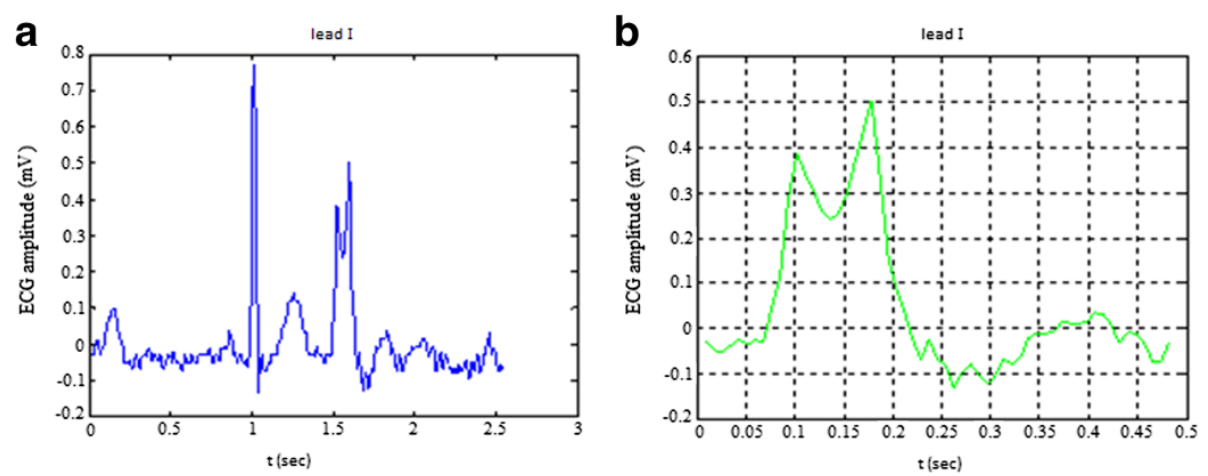

Figure 3 ECG waveforms of a patient with RVOT septum PVC in lead I (a) and its PVC detected (b). 
Table 1 The statistical analysis (p-values), one-way ANOVA, of the 12 leads ECG features between the five groups

\section{Table 1 The statistical analysis (p-values), one-way ANOVA, of the 12 leads ECG features between the five groups}

\begin{tabular}{|c|c|c|c|c|c|c|c|c|c|c|c|c|}
\hline Features leads & Lead I & Lead II & Lead III & Lead aVR & Lead aVL & Lead aVF & Lead V1 & Lead V2 & Lead V3 & Lead V4 & Lead V5 & Lead V6 \\
\hline QRS polarity & 0.019 & 0.001 & 0.001 & 0.001 & 0.001 & 0.001 & 0.001 & 0.001 & 0.001 & 0.001 & 0.001 & 0.001 \\
\hline Average of Haar wavelet coefficients at level 1 & 0.231 & 0.508 & 0.917 & 0.544 & 0.969 & 0.496 & 0.249 & 0.415 & 0.284 & 0.627 & 0.513 & 0.924 \\
\hline Average of Haar wavelet coefficients at level 2 & 0.67 & 0.559 & 0.588 & 0.580 & 0.096 & 0.101 & 0.795 & 0.582 & 0.556 & 0.725 & 0.135 & 0.564 \\
\hline Maximum amplitude of spectrum signals & 0.001 & 0.001 & 0.007 & 0.001 & 0.172 & 0.001 & 0.001 & 0.33 & 0.009 & 0.002 & 0.001 & 0.001 \\
\hline Variance of signals' spectrum & 0.002 & 0.001 & 0.010 & 0.001 & 0.254 & 0.001 & 0.001 & 0.037 & 0.036 & 0.001 & 0.001 & 0.001 \\
\hline Average of FFT of signal & 0.002 & 0.001 & 0.001 & 0.001 & 0.099 & 0.001 & 0.001 & 0.064 & 0.430 & 0.001 & 0.001 & 0.001 \\
\hline Power spectrum of signals & 0.002 & 0.001 & 0.010 & 0.001 & 0.193 & 0.001 & 0.002 & 0.031 & 0.037 & 0.002 & 0.002 & 0.001 \\
\hline Power spectrum band width of signals & 0.001 & 0.001 & 0.013 & 0.001 & 0.218 & 0.001 & 0.002 & 0.068 & 0.073 & 0.002 & 0.003 & 0.005 \\
\hline Average of amplitudes of spectrum signals greater than the 70 percent & 0.085 & 0.002 & 0.160 & 0.009 & 0.171 & 0.001 & 0.001 & 0.309 & 0.373 & 0.009 & 0.290 & 0.032 \\
\hline Average of spectrogram greater than the 70 percent & 0.001 & 0.001 & 0.009 & 0.001 & 0.175 & 0.001 & 0.001 & 0.035 & 0.005 & 0.002 & 0.001 & 0.001 \\
\hline
\end{tabular}

Using one-way ANOVA, the $p$-values of the 12 lead ECG features between five groups are shown in this table. 
Error examination revealed that the most error (obtained by using SVM classification) was associated with classification of both groups RVOT septum and aortic cusps (42.86\%), while the other two groups had less errors $(<15 \%)$ and rather appropriate separation results.

\section{Discussion}

This study shows a method to identify PVCs and determine their foci by ECG signals processing. This method was carried out on ECGs of 87 patients in five groups, LVOT, RVOT septum, basal RV, RVOT free-wall and aortic cusp, using wavelet, frequency, spectrogram analysis and morphological features.

The results of statistical analyses in this study demonstrated that wavelet features have no significant values while the morphological and frequency features have the most significant difference, respectively. The best classification result of $88.4 \%$ was obtained by considering all the features. Our findings also illustrate that classification of groups 2 and 5 (RVOT septum and aortic cusp region) was not precise (42.86\% error). This means that it's difficult to differentiate PVCs origins in the RVOT septum and aortic cusp region truly due to their close anatomic location. Therefore these features cannot sufficiently distinguish these two groups and it is required to use other features.

Most of researches in this regard have used some features to differentiate among foci, while in this study various features were evaluated. The features such as QRS amplitude ratio between leads aVR/aVL and II/III, time to earliest rapid deflection in precordial leads and interval to peak of $\mathrm{R}$ wave in lead V2 was used to predict an epicardial origin for LV-VT/PVCs by Bazan et al. (2007). Reported ECG morphologic features, including the presence or absence of a Q wave in leads II, III and aVF, were region specific to identify epicardial origin. We used more morphological features with the other features, spectrum and spectrogram, for localizing five groups.

Dixit et al. (2005) used QRS morphology, amplitude, and duration in precordial leads to categorize the origin of VT/PVCs in medial and lateral locations along basal $\mathrm{LV}$ region. It has been reported that using the ECG criteria was possible to localize the site of origin of clinical arrhythmia in $83 \%$ of idiopathic VT/PVC originating from basal LV, while in this study using more features, better classification was obtained $(88.4 \%)$ for five groups.

Our method to the best of our knowledge is the first to employ spectral content and spectrogram values of ECGs for classification of PVC beats and determining their foci. Based on the findings, it is concluded that use of surface ECG signals, a non-invasive method, is helpful to predict PVCs and their foci and will facilitate PVC/ VT localization. The proposed method can be important for ablation procedure and may help to improve ablation outcome. As a future work, it is suggested to explore more appropriate features in order to improve the result of the classification with more data set.

\section{Competing interests}

The authors declare that they have no competing interests.

\section{Authors' contributions}

SS carried out the data collection, signal processing consist of PVC detection, ECG processing, localization of premature ventricular contraction foci classifying of the five groups and also participated in the sequence alignment and drafted the manuscript. AS has performed some of proportions of signal processing algorithms such as features extraction and classifying and as an advisor for statistical analysis and also participated in the sequence alignment and drafted the manuscript. AGS has selected patients and performed Electrophysiology studies (EPS) and had have been super advisor scene on the obtained results. MMD carried out the data collection and signal preprocessing too. All authors read and approved the final manuscript.

\section{Acknowledgements}

This research has been supported by Islamic Azad University, Science and Research branch-Tehran-Iran, Tehran Arrhythmia Clinic and Day General Hospital. We wish to thank Mrs. Davoud Abadi and Mr. Manouchehr Ahmadvand for their helps.

\section{Author details}

1Department of Biomedical Engineering, Islamic Azad University, Science and Research branch, Tehran, Iran. ${ }^{2}$ Assistant Professor of Department of Biomedical Engineering, Islamic Azad University, Science and Research branch, Tehran, Iran. ${ }^{3}$ Cardiologist and Electrophysiologist of Tehran Arrhythmia Center, Tehran, Iran. ${ }^{4}$ Non-invasive cardiac electrophysiology research laboratory, Day general hospital, Tehran, Iran.

Received: 9 April 2013 Accepted: 9 September 2013 Published: 25 September 2013

\section{References}

Bazan V, Gerstenfeld EP, Garcia FC, Bala R, Rivas N, Dixit S, Zado E, Callans DJ, Marchlinski FE (2007) Site-specific twelve-lead ECG features to identify an epicardial origin for left ventricular tachycardia in the absence of myocardial infarction. Heart Rhythm Society 4:1403-1410

Bensegueni S, Bennia A (2012) Premature Ventricular Contractions Classification of QRS Complexes. Proceedings of MISC 2012 - 2nd International Symposium on Modeling and Implementation of Complex Systems Constantine. , Algeria

Betensky BP, Park RE, Marchlinski FE, et al. (2011) A new electrocardiographic criterion for distinguishing left from right ventricular outflow tract tachycardia origin FREE. J Am Coll Cardiol 57(22):2255-2262. doi:10.1016/j.jacc.2011.01.035

Chang RC-H, Lin C-H, Wei M-F, Lin K-H, Chen S-R (2013) High-precision real-time premature ventricular contraction (PVC) detection system based on wavelet transform. Springer Science+Business Media, New York. DOI 10.1007/ s11265-013-0823-6

Chikh MA, Ammar M, Marouf R (2010) A neuro -fuzzy identification of ECG beats. J Med Syst. Doi: 10.1007/s10916-010-9554-4

Chiu CC, Lin TH, Liau BY (2005) Using correlation coefficient in ECG waveform for arrhythmia detection. Biomed Eng App, Bas C 17:147-152

Dixit S, Gerstenfeld EP, Lin D, Callans DJ, Hsia HH, Nayak HM, Zado E, Marchlinski FE (2005) Identification of distinct electrocardiographic patterns from the basal left ventricle: distinguishing medial and lateral sites of origin in patients with idiopathic ventricular tachycardia. Heart Rhythm Society 2:485-491

Hardalac F, Yildirin H, Serchatlioglu S (2007) Determination of arotic disease with the application of STFT and CWT methods. Comput Biol Med 37:785-792

Kamakura S, Shimizu W, Matsuo K, Taguchi A, Suyama K, Kurita T, Aihara N, Ohe T, Shimomura K (1998) Localization of optimal ablation site of idiopathic ventricular tachycardia from right and left ventricular outflow tract by body surface ECG. (c) 1998 Am Heart Assoc 98:1525-1533. Inc.Circulation1998

Lerman BB, Stein KM, Markowitz SM (1997) Mechanism of idiopathic left ventricular tachycardia. J Cardiovasc Electrophysiol 8(5):571-583

Lin D, Ilkhanoff L, Gerstenfeld E, Dixit S, Beldner S, Bala R, Garcia F, Callans D, Marchlinski FE (2008) Twelve-lead electrocardiographic characteristics of the 
aortic cusp region guided by intracardiac echocardiography and electroanatomic mapping. Heart Rhythm Society 5:663-669

Martínez JP, Almeida R, Olmos S, Rocha AP, Laguna P (2004) A wavelet-based ECG delineator: evaluation on standard databases. IEEE T Bio-Med Eng 51(4):570-581

Nakagawa M, Takahashi N, Nobe S, et al. (2002) Gender differences in various types of idiopathic ventricular tachycardia. J Cardiovasc Electrophysiol 13:633-638

Nathani P, Shetty S, Lokhandwala Y (2007) Ventricular tachycardia in structurally normal hearts: recognition and management. Supplement of J Assoc Physicians India 55(suppl):33-38

Sheikkani A, Behnam H, Mohammadi MR, Noroozian M, Mohammadi M (2012) Detection of abnormalities for diagnosing of children with asperger disorders using of quantitative electroencephalography analysis. J Med Syst 36:957-963

Shin SY, Joo HJ, Kim JH, Jang JK, Park JS, Kim YH, Lee HS, Choi JI, Lim HE, Kim YH (2007) Epicardial conduction properties and electrocardiographic characteristics of premature ventricular complexes or ventricular tachycardias that originate at the aortic cusp. Korean Circ J 37:616-622

Song MH, Lee J, Cho SP, Lee KJ, Yoo SK (2005) Support vector machine based arrhythmia classification using reduced features. Int J Control Autom 3(4):571-579

Subha DP, Joseph PK, Achary UR, Lim CM (2010) EEG signal analysis: a survey. J Med Syst 34:195-212

Sumathi S, Sanavullah MY (2009) Comparative study of QRS complex detection in ECG based on discrete wavelet transform. Int J Recent Trends Eng 2(5):273-277

Tada H, Tadokoro K, Ito S, Naito S, Hashimoto T, Kaseno K, Miyaji K, Sugiyasu A, Tsuchiya T, Kutsumi Y, Nogami A, Oshima S, Taniguchi K (2007) Idiopathic ventricular arrhythmias originating from the tricuspid annulus: prevalence, electrocardiographic characteristics, and results of radiofrequency catheter ablation. Heart Rhythm Society 4(1):7-16

Tokmakei M, Erdogan N (2009) Investigation of the arterial stiffness on renal artery Doppler sonograms. J Med Syst 33:101-106

Yamashina Y, Yagi T, Namekawa A, Ishida A, Sato H, Nakagawa T, Sakuramoto M, Sato E, Yambe T (2011) Prevalence and characteristics of idiopathic right ventricular outflow tract arrhythmias associated with J-waves. Clin Res Electrophysiology Ablation Europace 13:1774-1780. doi: 10.1093/europace/ eur256, 2011

Yeh YC (2009) An analysis of ECG beats by using the mahalanobis distance method. IEEE Computer Society, Washington, DC, USA. Doi: 10.1109/ ICICIC.2009.75

doi:10.1186/2193-1801-2-486

Cite this article as: Soheilykhah et al.: Localization of premature ventricular contraction foci in normal individuals based on multichannel electrocardiogram signals processing. SpringerPlus 2013 2:486.

\section{Submit your manuscript to a SpringerOpen ${ }^{\circ}$ journal and benefit from:}

- Convenient online submission

- Rigorous peer review

- Immediate publication on acceptance

- Open access: articles freely available online

- High visibility within the field

- Retaining the copyright to your article

Submit your next manuscript at $>$ springeropen.com 